\title{
Albrecht von Haller, Gerard van Swieten und Boerhaavens Erbe
}

\author{
Von ERna Lesky
}

Die Beziehungen Albrecht von Hallers (1708-1777) zu den Häuptern der Wiener Schule standen unter keinem guten Stern. Von dem Streit des Klinikers Anton de Haen (1704-1776) mit Haller um die Irritabilitätslehre kann man manches - zum Teil sogar über dem Strich - in den älteren medizinhistorischen Handbüchern lesen ${ }^{1}$. Dann gab es aber auch eine Spannung zwischen Haller und van Swieten (1700-1772), die Zeit ihres Lebens wegen einer Äußerung des Hannoverschen Leibarztes und Haller-Freundes Paul Gottlieb Werlhof (1699-1767) über ihre jeweils verschiedene Art, Boerhaave zu kommentieren, bestanden haben soll. Diesen Vermerk findet man nur mehr unter dem Strich ${ }^{2}$ mit dem Hinweis auf die ZimmermanNsche Haller-Biographie des Jahres $1755^{3}$. Hier lohnt es sich weiter zu fragen: Was kann die beiden größten Boerhaave-Schüler, die Erben Boerhaaves schlechtweg, ein ganzes Leben so getrennt haben? War es wirklich nur der Umstand, daß der eine, Haller, seinen Kommentar vom Boerhaave-Text reinlich trennte, der andere aber, van Swieten, beides vermischte? Oder birgt sich hinter dem Formalen nicht eine grundsätzlich verschiedene Auffassung vom Pietätsverhältnis zum Lehrer, zu Boerhaave? Und spiegelt sich in dieser Verschiedenheit nicht eine ebensolche von Wesen und Weg wissenschaftlicher Forschung? Diese Fragen an einem bisher noch nicht herangezogenen Quellenmaterial zu klären und dadurch zwei grundverschiedene Forscherpersönlichkeiten hervortreten zu lassen, ist die Aufgabe, die sich diese Untersuchung stellt.

Herman BoerhaAve (1668-1738) war eine faszinierende Lehrerpersönlichkeit gewesen ${ }^{4}$. An 2000 europäische Ärzte hatten dies erfahren, und

1 J.F.C.Hecker, Geschichte der neueren Heilkunde, Berlin 1839, S. 415 ff. H.HAESER, Lehrbuch der Geschichte der Medicin und der epidemischen Krankheiten, 2. Band, Jena 1881 , S. 581.

2 E.G.Baldinger, Biographien jetztlebender Ärzte und Naturforscher, 1.Band, Jena 1772, S. 17, Anmerkung r. J.F.C.Hecker, a.a.O., S. 391, Anmerkung 2. L. Hirzel, Albrecht von Hallers Gedichte, Frauenfeld 1882, S. CCLIII, Anmerkung 2. M.NeubUrger, Das alte medizinische Wien in zeitgenössischen Schilderungen, Wien und Leipzig 1921, S. 11, Anmerkung 2.

3 J.G.Zimmermann, Das Leben des Herrn von Haller, Zürich 1755, S. $220-5$.

${ }^{4}$ Dazu vgl. man die umfassenden Würdigungen bei P.DiePgeN, Hermann Boerhaave und die Medizin seiner Zeit mit besonderer Berücksichtigung seiner Wirkung nach 
Haller nennt ihn mit Recht «communis Europae sub initio huius saeculi magister». Dieser säkulare Lehrer hatte aber von seiner Lehre verhältnismäßig wenig in Druck gegeben und dieses in der aphoristischen Kürze des Hippokrates, als dessen Erbe er sich fühlte. Mehr und alles von ihm zu besitzen, war der lebhaft und allgemein gefühlte Wunsch, dem geschäftstüchtige Verleger nachkamen, indem sie schlechte und fehlerhafte Vorlesungsnachschriften nicht genannter Schüler überall auf den Markt brachten, in Padua, Venedig und anderswo.

Dieser Unfug griff so um sich, daß der Meister selbst in der Leidener Zeitung vom 9. Oktober 1726 dazu öffentlich und empört Stellung nahm ${ }^{5}$ und im Vorwort zu seinen 1728 erschienenen Elementa chymiae eine Liste jener Drucke bekanntgab, die er als autorisiert anerkannte ${ }^{6}$. Wie sehr sich der rechtlich denkende Mann und gütige Lehrer beleidigt und betrogen fühlte, kann man daraus ermessen, daß ex im gleichen Jahre, 1728, auch von der Niederländischen Regierung das Verbot des Nachdruckes von Werken Leidener Professoren erwirkte ${ }^{7}$. Seit dieser Zeit galt die Veröffentlichung von Boerhaave-Vorlesungen in der wissenschaftlichen Welt und besonders im engeren Schülerkreis um den Meister als diskriminierend.

Dieses Vorspiel muß man in Rechnung setzen, wenn man einerseits verstehen will, daß sich jetzt nach dem Tode des Meisters 1738 selbst so prominente Schüler wie Haller und van Swieten nur widerstrebend entschlossen, den Bitten von Verlegern und Freunden zu willfahren und ihre Vorlesungsnachschriften bzw. Kommentare herauszugeben, und anderseits, daß der dabei jeweils von ihnen gewählten Form eine bisher nicht beachtete Bedeutung zukommt.

Deutschland, Hippokrates 10 (1939) 298-306, 345-51. Derselbe, Der Weg von Boerhaavens Medizin nach Deutschland, Memorialia Herman Boerhaave opt. med. Haarlem 1939, S. 23-31. H.E.Sigerist, Große Ärzte, München 1954, S. 163-70. Ferner: M. NEUBURGER, Boerhaaves Einfluß auf die Entwicklung der Medizin in Österreich, Janus 23 (1918) 215-22.

5 Abgedruckt bei E.Cohen, Herman Boerhaave und seine Bedeutung für die Chemie, Janus 23 (1918) 241; desgleichen bei E. C.van Leersum, Cours de Boerhaave, en particulier ses leçons cliniques, Janus 23 (1918) 319f.

${ }^{6}$ Dazu vgl. die Ausführungen in der Bibliothèque Britannique ou Histoire des ouvrages des sçavans de la Grand-Bretagne 24 (1746) $189 \mathrm{ff}$.; ferner E.C.van LeERSuM, a.a.O., $318 \mathrm{f}$.

7 Darüber lese man den Bericht seines Kollegen, des Leidener Anatomen BernHard SiegFrIEd Albinus, Annotationes academicae, Lugd. Bat. 1754, Lib. III, Ad cap. XII, p. 84 ss.; die Verordnung selbst ist abgedruckt in Bibliothèque Britannique 24 (1746) 194f. 
Haller begann 1739 mit der Veröffentlichung seiner Vorlesungsmitschriften, und zwar des theoretischen Teils der Boerhaaveschen Lehre (Anatomie, Physiologie, allgemeine Krankheitslehre). Er tat es unter dem Namen des Lehrers selbst: Hermanni Boerhaave Praelectiones academicae in proprias institutiones rei medicae edidit, et notas addidit Albertus Haller ${ }^{8}$. Dabei verfuhr er so, daß er seinen eigenen Kommentar klar und deutlich von dem Boerhaave-Text trennte, indem er jenen nicht nur unter den Strich setzte und einrückte, sondern auch durch den Petit-Druck abhob. Im Vorwort zum vierten, 1743 erschienenen Band ${ }^{9}$ äußert er sich auch über den Grund, der ihn diese Form wählen ließ: «... damit nicht jemand dem Meister meine Lehren oder Fehler, die nicht die seinen sind, zurechne.»

Tatsächlich hatte nämlich Haller eine Menge Eigenes in den Kommentar hineingesteckt, im ganzen nicht mehr und nicht weniger als fünfzehn Jahre ${ }^{10}$ Forschungsarbeit und soviel, daß aus dem einen Band der Boerhaaveschen Originalausgabe bei Haller sieben Bände wurden ${ }^{11}$. Eine Fülle von eigenen anatomischen Erkenntnissen und experimentellen Beobachtungen ist hier vielfach erstmals niedergelegt oder zumindest angebahnt, wie beispielsweise der für das spätere Forschungswerk so zentrale Begriff der Irritabilität ${ }^{12}$ und mit der ganzen enzyklopädischen Souveränität Hallerscher Literaturkenntnis verarbeitet. Denn diese ebenso wie sein Forschertemperament hatten den Kommentator über die ihm gestellte, rein dem Boerhaaveschen Text dienende Aufgabe fortgerissen: «Während ich mich mit dem Werke

8 Diese erste Ausgabe, die im folgenden benutzt wurde, erschien bei Abram Vandenhoek in Göttingen und fand mit dem Erscheinen des 6. Bandes 1744 ihren Abschluß. Boerhaave selbst hatte seine Institutiones medicae in usus annuae exercitationis domesticos digestae erstmals 1708 und dann 1713, 1720,1727 und 1734 in vermehrter Auflage herausgebracht. Über weitere Ausgaben und U̇bersetzungen vgl. J. F. FuLtoN, The Influence of Boerhaave's Institutiones medicae on Modern Physiology, Memorialia H. Boerhaave, Haarlem 1939, S. 60-6.

91742 war inzwischen der erste Band der anders angelegten Ausgabe van Swietens erschienen!

10 Dazu vgl. J.G.Zimmermann, a.a.O., S. 175; ferner die Vorrede zum 1. Band der Elementa physiologiae corporis humani, die im folgenden in der deutschen Übersetzung Johann Samuel Hallen, Anfangsgründe der Phisiologie des menschlichen Körpers, Berlin 1759-1776, zitiert werden.

11 Der fünfte Band umfaßt zwei Teile.

12 In der Annot. i (Prael. § 87, p. 129) berichtet HALler, daß herausgeschnittene Herzen von Kaltblütern noch stundenlang pulsieren. Daran schließt er die Folgerung: «Ergo cor movetur a causa aliqua, quae neque a cerebro neque ab arteriis derivatur, ignota et in ipsa fabrica cordis latente." 
beschäftigte und, wie es zu geschehen pflegt, dabei warm wurde, befiel mich eine unwiderstehliche Leidenschaft (ardor), den Beschreibungen der Teile das hinzuzufügen, was durch das eigene Experiment wahr erschien oder aus Autoren von sicherer Glaubwürdigkeit geholt werden konnte, die Boerhaave durch seine vielen Geschäfte oder aus Rücksicht auf seine Zeit nicht aufgeschlagen hatte. Also schwoll das Buch sowohl an Umfang als weitmehr noch durch die Schwierigkeit der Aufgabe an, da ich allmählich zum Autor wurde, der ich mich als Herausgeber bekannt hatte ${ }^{13}$.

Kein Wunder, daß der Autor unter dem Strich mit dem Autor über dem Strich nicht immer übereinstimmte und das schon im Oktober 1730, als Haller, mit der Kommentierung des Kapitels «Kau- und Schluckorgane» (deglutitio) befaßt, offen seinem Freunde, dem Zürcher Kanonikus JohanNes GeSner (1709-1790), bekennt: «... in quibus ab auctore (sc. Boerhaavio) totus differo ${ }^{14}$.

Es läßt sich nun an den einzelnen Bänden der Praelectiones verfolgen, wie die Kommentierung der Boerhaaveschen Lehre für Haller bei allem immer wieder geäußerten Respekt vor dem Lehrer stellenweise zu einer intensiven Auseinandersetzung mit ihm wird, aus der die eigene Lehrmeinung herauswächst, sich zu klären und zu verselbständigen beginnt, so daß sie oft exkursartig den Boerhaave-Text überwuchert und über Seiten hinweg geradezu ein Eigenleben führt. Das kann man besonders in den Annotationen zur Gefäßlehre der Boerhaaveschen Institutiones von 1734 beobachten. In strenger Objektivität, oft ohne den Namen des Lehrers überhaupt zu nennen, werden dessen Ansichten von der Gefäßversorgung des Magens und der Eingeweide auf Grund der besseren Literaturkenntnis

13 In der Dedicatio des ersten Bandes der Praelectiones.

14 H.E.Sigerist, Albrecht von Hallers Briefe an Johannes Gesner (1728-1777). Abh. Ges. Wiss. Göttingen, Math.-phys. Klasse, N.F. XI/2, Berlin 1923 (im folgenden zitiert als Sigerist). Brief Nr. 6, S. 25. Im einzelnen vgl. den Widerspruch Hallers in der Annot. $28 \mathrm{zu}$ der Ausgabe der Boerhaaveschen Institutionen von 1734 (Prael. § 70, p. 173) über die Funktion des M. azygos = Gaumensegelmuskel. - Weitere kritische Bemerkungen Hallers über Boerhaave finden sich auch in den folgenden Jahren in den Briefen an Gesner, so beispielsweise 1734 im Zusammenhang mit der Beschreibung der Umbelliferen: «Quam negligenter has noster Boerhaavius ?» (Sigerist, Nr. 53, p. 99) oder 1735 in bezug auf die Klassifizierung von Pflanzen: «Nonne ergo plurima emendanda in methodo praeceptoris nostri?» (Sigerist, Nr. 65, p. 111). Diese bereits in den dreißiger Jahren eintretende Distanzierung Hallers von Boerhaaveschen Lehrmeinungen hat bereits ANNA Ischer, Albrecht von Haller und das klassische Altertum. Sprache und Dichtung, Forsch. Sprach. u. Literaturwiss, H. 41, Bern 1928, S. 24.f. beobachtet und hervorgehoben. Vgl. auch P.DiEPGEN, Memorialia ..., S. 28. 
Hallers und der inzwischen und zum Teil von ihm selbst gefundenen neuen Tatsachen außer Kurs gesetzt ${ }^{15}$. Als überholt stellt sich ebenfalls Boerhaaves Lehre vom Wandbau des Magens dar ${ }^{16}$, und als unhaltbar muß unter anderen Lehrmeinungen Boerhaaves auch jene über die Einteilung der Gefäße in Blut-, seröse und lymphatische erwiesen werden ${ }^{17}$. Gewiß, ein «elegans systema» im Sinne der Zeit, die es liebte, willkürliche Spekulationen mechanistischer Art als Lehrdogma zu setzen wie hier: daß ein jeweils immer kleiner werdender Durchmesser verschiedener flüssiger Einheiten wie der Blut-, Serum- und Lymphglobuli eine ebensolche Verkleinerung der verschiedenen Gefäßlumina bedinge. In seiner Argumentation gegen diese Hypothese Boerhaaves muß Haller den Namen ihres Schöpfers, den Namen seines Lehrers, nennen. Aber deshalb bleibt dieser doch immer der "praeceptor optimus», auch wenn der Schüler von ihm abweicht, abweichen muß zufolge des leidenschaftlichen Forscherdranges, der eben diesen Schüler zwingt, überall neue Bahnen aufzureißen und, den Lehrer korrigierend, über diesen hinauszuschreiten. So wird dieser Schüler gerade in der Auseinandersetzung mit dem Werk des Meisters zum künftigen Autor der Elementa physiologiae und damit zum Meister selbst. Haller hat stets und besonders in der Vorrede zum ersten Band des letztgenannten Werkes bekannt, was für ihn die Arbeit an den Boerhaave-Kommentaren bedeutete: die hohe Schule der Physiologie, den entscheidenden Schritt zur Vollendung. Sind so die Elementa das Werk der Reife geworden, so kommt doch ihrer Vorstufe, den Kommentaren, der Ehrentitel zu, die erste «histoire exacte du corps humain vivant» zu sein, «un peu hérissé et moins à la portée de tout le monde ${ }^{18}$, weil überquellend in noch nicht gebändigter Gelehrsamkeit ${ }^{19}$.

${ }^{15}$ So beispielsweise Prael. § 77, p. 214, Annot. 14 ss., wo HALler zwar noch als Norm an der Zweiteilung der A. coeliaca in A. hepatica und splenica, aus welcher die A. coronaria vel gastrica sup. (= A. gastrica sin.) entspringe, festhält; aber als Varietät nach WINSLow vermerkt er bereits über den Ursprung der letzten, daß sie auch «bisweilen aus dem Stamm der A. coeliaca selbst hervorgeht, welche dann gleichsam ,tripes ${ }^{6}$ erscheint ». Den nach HaLler benannten Tripus der A. coeliaca als ihre normale Verzweigungsform hat sein Entdecker bekanntlich im zweiten Faszikel der Iconum anatomicarum partium corporis humani 1745 dargestellt.

16 Prael. § 81, p. 232, Annot. 3.

17 Prael. § 245, p. 407, Annot. c.

18 So charakterisiert in Bibliothèque raisonnée des ouvrages des savans de l'Europe 40 (1748) 467.

19 Trotzdem hatte das Werk einen großen Erfolg: 1740 erschien der erste Band bereits in zweiter Auflage; es wurde in Turin, Venedig, Altdorf nachgedruckt und ins Englische 
Aber Haller begnügte sich nicht mit der Edition des theoretischen Teils der Boerhaaveschen Lehre. Wir wissen aus dem Vorwort zum ersten Band der Praelectiones, daß er bereits damals, 1739, den Plan gefaßt hatte, auch den praktischen Teil, die Aphorismen, herauszugeben. Hierin aber kam ihm Gerard van Swieten 1742 zuvor. So ist es wohl nicht allein eigener Entschluß, «diese Sparte» van Swieten «abzutreten», dessen Tätigkeit mit der Praxis aufs engste verbunden $\mathrm{sei}^{20}$, als die notwendige Konsequenz aus dem von van Swieten gesetzten Faktum.

Als Gerard van Swieten bei der Veröffentlichung «dieser Sparte», des praktischen Teils der Boerhaaveschen Lehre, seinen Namen mit dem seines Lehrers verband, da war er noch praktischer Arzt in Leiden ${ }^{21}$. Dies ins Auge zu fassen, ist wichtig. Denn aus diesem Umstand läßt sich die bisher überhaupt nicht beachtete Verschiedenheit der Editionsform Hallers und der van Swietens auch erklären. Was nämlich für den Göttinger Professor nicht galt, das holländische Nachdruckverbot für Boerhaave-Vorlesungen aus dem Jahre 1728, für den Leidener Arzt und Bürger war es verpflichtend. van Swieten trug diesem Umstand Rechnung, indem er nicht wie Haller seine Vorlesungsmitschriften unter dem Namen des Lehrers herausgab, sondern auf Grund dieser einen Kommentar verfaßte und diesen unter seinen Namen als den Autornamen stellte: Gerardi van Swieten Commentaria in Hermanni Boerhaave aphorismos de cognoscendis et curandis morbis ${ }^{22}$.

Und tatsächlich bildet ja sein Kommentar anders als bei Haller den Hauptbestandteil des Textes. Während nämlich Hallers Kommentar als in Petit gedruckte, mit Zitaten und gelehrter Kritik überladene Anmerkung unter dem Strich erscheint, fließt der van Swietensche Kommentar, in etwas kleinerem Druck von dem Aphorismus Boerhaaves abgehoben, in einer kontinuierlichen, leicht les- und faßbaren, den Lehren des Meisters Wort für Wort folgenden Paraphrase dahin. Denn der Verfasser dieser Para-

und Französische übersetzt. Ja, J.G.ZrmmermanN, a.a. O., S. 174, behauptet, daß «von dieser Zeit an (der) blühende Zustand der Vanderhoekischen Handlung » in Göttingen datiere.

20 A.v.Haller, Bibliotheca med.pract., t. IV, Basel 1788, p. 152: Continuassem ast cum audivi, Gerhardum a Swieten commentarios in Aphorismos promittere, meum statui ei hanc spartam cedere, cujus vita in praxi occupatur.

${ }^{21}$ Erst 1745 folgte van Swieten dem Rufe Maria Theresias nach Wien.

${ }^{22}$ Die erste Ausgabe erschien in fünf Bänden, Lugd. Bat. 1742-1772. Wir benutzten die zweite Ausgabe, Lugd. Bat. 1745. Das Werk wurde außerdem noch aufgelegt in Paris, Turin, Venedig und Hildburghausen und bereits 1747 ins Englische und Französische übersetzt. Vgl. E.G.BALDINGER, a.a. O., S. 14f. 
phrase will nicht neue Fakten bringen und durch sie diejenigen des Lehrers ergänzen oder gar kritisieren; er will nicht mehr sein als der getreue Interpret der Lehren seines Meisters, mit denen er sich - wenigstens in den hier zur Debatte stehenden zwei ersten, 1742 und 1745 erschienenen Bänden seines Werkes - bis in die letzten Details des Boerhaaveschen Systems identifiziert.

War doch Boerhaave für van Swieten das «Orakel» ${ }^{23}$ schlechtweg, und Geist und Lehren dieses einzigartigen Mannes der Nachwelt zu überliefern, die Aufgabe, die zu erfüllen, er sich durch sein zwanzigjähriges Schülerverhältnis berufen fühlte. Davon zeugt die Widmung seiner Kommentare in der doppelten Form des Titelbildes und der zueignenden Worte am Ende der Praefatio. Beides will mehr sein als die übliche Dedikation der Zeit wie etwa bei Haller, der die Bände seiner Kommentare Freunden widmet, den ersten Werlhof, den zweiten van der Laar, den dritten Gesner usf.; van Swietens Widmung bedeutet ein Programm: die Sanktifizierung und Äternisierung Boerhaaveschen Geistes schlechtweg. Das ist der Sinn des Titelbildes mit der Grabstele Boerhaaves in der Peterskirche zu Leiden und der Inschrift darauf: salutifero Boerhaavii genio sacrum, und ebenso der Schlußworte der Dedikation: Immortali memoriae magni Boerhaavii.

Kein Wort dieses großen Mannes zu verlieren, sondern jedes aufzuschreiben und der Nachwelt treu zu bewahren, das hatte sich schon der junge van Swieten zur Aufgabe gesetzt, dem Haller 1725 in Leiden begegnet war und über den er in sein Tagebuch ${ }^{24}$ anmerkte: «... der Krafft einer charakteren Schrift alle Sachen von Boerhaave von Wort zu Wort abgeschrieben. Ein gescheuter Mensch...» Mit der «charakteren Schrift» meint Haller die Ramsaysche Tachygraphie, die schon der 24jährige van Swieten, wie der Entzifferer seiner Schnellschrift, E.C.van Leersum ${ }^{25}$, feststellte, meisterlich beherrschte. Zufolge dieser Meisterschaft konnte er alle BoerhaaveVorlesungen der Jahre 1724 bis 1737/1738 wortwörtlich in Vorlesungsmitschriften festhalten und schuf sich damit die Grundlage nicht nur für seine Kommentare, sondern auch für seine späteren Vorlesungen über die Institutionen im Vorsaal der Wiener Hofbibliothek, die diesen größtenteils

23 Comment., Praef.: «Probe enim memini, et gratus recordor, me nunquam accessisse hoc oraculum, quin redierim doctior.»

24 L. Hirzel, Albrecht von Hallers Tagebücher seiner Reisen nach Deutschland, Holland und England. 1723-1727, Leipzig 1883, S. 110. E. Hintzsches Ausgabe, St. Gallen 1948, war mir nicht zugänglich.

25 E. C.van Leersum (siehe Anmerkung 5) S. 323. 
noch nicht entzifferten Schatz heute noch aufbewahrt ${ }^{26}$. Durch ihn war van Swieten wie kein anderer Boerhaave-Schüler befähigt und fühlte sich eben durch sein zwanzigjähriges Schülerverhältnis «forte unico » ${ }^{27}$ auch berufen, nicht nur Editor und Kommentator, sondern auch der Konservator des Boerhaaveschen Erbes zu sein.

Dieses Erbe konnte man aber nur konservieren, wenn man die Lehre nicht nur in möglichst reiner, sondern auch in möglichst einfacher und faßbarer Form durch den Druck weitergab. Ja, diese Art der Weitergabe war für van Swieten als einzige und daher als zwingend notwendige übriggeblieben, als man ihm 1738 nach dem Tode Boerhaavens wegen seines katholischen Glaubensbekenntnisses die Fortführung seiner Vorlesungen an der protestantischen Universität Leiden untersagte. Der klar und leicht faßlich geschriebene Kommentar mußte also den mündlichen Vortrag ersetzen. Und das hatte van Swieten zunächst auch noch 1749 in Wien im Auge, als er Maria Theresia seinen Reformplan für das medizinische Studium vorlegte und hinsichtlich von Kollegs der praktischen Heilkunde äußerte ${ }^{28}$ : «Cet ouvrage (sc. seine Kommentare) remplira parfaitment mon dessein et on aura pas besoin de donner alors un collège sur cette partie de la medecine. car je crois m'avoir expliquer si clairement que les estudiants seront tous en estat de le comprendre, après qu'ils auront assisté deux ans au lecons que je donne sur les Institutions de la medecine.»Wenn er dann ab 1754 seinen Freund und Leidener Studienkollegen Anton de Haen auch ein Kolleg über den praktischen Teil lesen ließ, so ist doch sein Werk, die Kommentare, das Lehrbuch der praktischen Medizin geblieben in Wien sogar bis zum Jahrhundertende -, allgemein anerkannt und geschätzt «par sa facilité et par le bon usage qu'il a fait des Observations des plus grands Maitres».

Das hat sogar der anonyme Kritiker der Bibliothèque raisonnée ${ }^{29}$ anerkannt, einer bei J.Wetstein in Amsterdam erscheinenden Rezensierzeitschrift, in der er die Hallerschen Boerhaave-Kommentare anzeigte, anonym, wie es in dieser Zeitschrift üblich war. Bei dieser Gelegenheit läßt er sich zu einem Vergleich der Werke beider Boerhaave-Schüler verlocken

26 E. C.van Leersum gibt in einer Appendix seiner in Anmerkung 5 zitierten Arbeit S. 335-46 eine Beschreibung dieser Sammlung der Österreichischen Nationalbibliothek.

27 Comment., Praef.: «Rara certe felicitate mihi contigit, forte unico, viginti fere annorum spatio MAGNI BOERHAAVII institutionibus frui, tam publicis, quam privatis ...»

${ }^{28}$ R.KınK, Geschichte der kaiserlichen Universität zu Wien, Band I/2, Wien 1854, S. 257.

29 Bibl. rais. 33 (1744) 45. 
und setzt an dem van Swietenschen aus, daß sein Autor eigene Zusätze nicht als solche kenntlich gemacht, sondern im Gegensatz zu Haller seine und des Lehrers Meinung vermischt bringe. Im übrigen, so fügt er hinzu, habe ein geistvoller Mann eine andere Betrachtung angestellt ${ }^{30}$ : «Mr. van Swieten, attaché inséparablement à son Maitre, en reçoit tous les Systèmes et toutes les Hypothèses. Mr. Haller, plein de vénération pour le même Maitre, n'en admet pourtant que ce qui lui paroit vrai, et il s'oppose, quoiqu'avec respect, au moindre faux brillant, qui pourroit l'éblouir. C'est, disoit-il, que Mr. van Swieten commente en Catholique, et Mr. Haller en Protestant.»

Den homme d'esprit, der dem Vergleich der beiden Boerhaave-Schüler eine solch epigrammatische Zuspitzung zu geben wußte, nannte Haller später in seinem Brief an van Swieten vom 26. Mai $1748^{31}$ «felicissimus medicus et mihi optime notus». Es ist kein anderer als sein Freund Werlhof, dem er den ersten Band der Praelectiones gewidmet hatte. Sein eben ausgeschriebenes Urteil war es nun, das eine tiefe Kluft zwischen den beiden größten Boerhaave-Schülern aufriß, indem es ihre tatsächlich verschieden aufgefaßte und geäußerte pietas dem Lehrer gegenüber als ein Problem von grundsätzlicher Bedeutung in aller Schärfe hervortreten und zu einem Konflikt innerhalb der Schule selbst werden ließ.

Man kann diesen in seiner ganzen Tragweite nur dann übersehen, wenn man sich mit noch weiteren Einzelheiten aus der Anzeige des Autors der Bibliothèque raisonée ${ }^{32}$ bekanntmacht. Im Grund stellt dieser zunächst nichts anderes fest, als was Haller bereits in der Vorrede zum ersten Band der Praelectiones hinsichtlich Boerhaaves angemerkt und der Befund an den Boerhaave-Kommentaren Hallers selbst bestätigt hatte: daß Haller sich nicht mit der Boerhaaveschen Anatomie begnügte, insonderheit nicht mit ihren Gefäß- und Nervenkenntnissen. Aber der Anonymus fügt noch ein Weiteres, gleichsam entschuldigend, hinzu: der große Mann sei eben in der Anatomie Autodidakt gewesen, habe vielfach überhaupt keine autoptischen Kenntnisse besessen, sondern sich genötigt gefühlt, irgendwelche Autoren abzuschreiben. Außerdem habe er ein System zu verteidigen gehabt, die Tatsachen also nicht so genommen, wie sie die Natur darbiete, sondern wie es seine Lehre verlange, während Herr Haller, unbelastet durch ein System, den Tatsachen unvoreingenommen gegenübertrete.

30 Bibl. rais. 33 (1744) 46.

31 Abgedruckt von E.G.Baldinger, Neues Magazin für Ärzte, 2.Band, Leipzig 1780, 3. St, S. 208.

32 Bibl. rais. 33 (1744) 33-50. 
Boerhaave, der geliebte Lehrer, erschien nun als ein «anatomes imperitus, et hypothesium conditor et ornator»! Bevor van Swieten 1748 mit dieser scharfen Formulierung ${ }^{33}$ das Boerhaave-Bild des Anonymus charakterisierte und in Acht und Bann tat, brach bereits der Sturm los. Ein Leidener Arzt und Boerhaave-Schüler, William N. Noortwyck ${ }^{34}$, war es, der sich als Verteidiger seines Lehrers und van Swietens berufen fühlte. Er ließ 1746 zwei Gegenartikel in der Bibliothèque Britannique ${ }^{35}$ erscheinen, in denen er Punkt für Punkt die Behauptungen des Autors der Bibliothèque raisonée zu widerlegen suchte. Man ist im 18. Jahrhundert bei solchen gelehrten Streiten wahrlich nicht sanft miteinander umgegangen und hat sich über das Sachliche hinaus viel persönlich Verletzendes an den Kopf geworfen. Haller sollte dies mehrfach erfahren so in dem bekannten, im selben Jahre 1746 beginnenden Streit mit dem Jenenser Jatrophysiker Georg Erhard Hamberger (1697-1755) ${ }^{36}$ wegen des Atmungsmechanismus, dann von seiten seines einstigen Anatomielehrers in Leiden, Bernhard SiegFried Albinus (1697-1770), später von Anton de Haen hinsichtlich der Irritabilitätslehre, von Gunz u. a. Mit einer gewissen Resignation stellt er daher 1763 in der Vorrede zum fünften Band der Elementa ${ }^{37}$ fest: «Mein Schicksal verhängt es, $\ldots$ ob ich gleich allen Gelehrten wohl will, und ihren Namen, sie mögen von mir halten, was sie wollen, niemals zu verkleinern suche; ... daß ich demohngeachtet doch ... fast Jahr vor Jahr bittre Schmähschriften wider mich erscheinen sehen mus.»

Diejenige Noortwycks war besonders schlimm, und es hat Haller tief verletzt, daß er in ihr den Fleiß von fünfzehn Gelehrtenjahren, seine Boerhaave-Kommentare, dem von Boerhaave seinerzeit verurteilten anonymen Machwerk gleichgesetzt sehen mußte. Mit einer solchen Art der Verteidigung hat Noortwyck am wenigsten van Swieten einen Dienst erwiesen, diesen vielmehr belastet. Denn Noortwyck stand ihm sehr nahe, er

33 E. G.Baldinger, Neues Magazin für Ärzte, 2. Band, Leipzig 1780. 3. St., S. 210.

${ }^{34} \mathrm{Er}$ hatte 1735 mit einer Dissertation De natura humana bei Boerhaave promoviert. Dazu vgl. J.E.Kroon, Boerhaave as Professor-Promotor, Janus 23 (1918) 309. 1743 war seine, im Kapitel «De conceptu» der Praelctiones (t. V, p. II, cap. DCLXVIII, p. 97 ss.) von HALLER oft zitierte Untersuchung Historia uteri gravidi in Leiden erschienen.

35 Bibliothèque Britannique 23 (1746) 336-67; 24 (1746) 187-216.

${ }^{36}$ Im einzelnen vgl. H. BuEss, Ein Physiologenstreit vor 200 Jahren, Experientia 2 (1946) 150 f. Dazu: H. Winterstein, ebenda 2 (1946) 380; W. BreDnow, Jena und Göttingen. Medizinische Beziehungen im 18. und 19. Jahrhundert, Jena 1949; P.DIEPGEN, Geschichte der Medizin, 2/1, Berlin 1951, S. 20.

37 Vorrede, S. III $\mathrm{f}$. 
war nämlich sein Schwager. Van Swieten hat sich denn auch von dem Libell Noortwycks völlig distanziert, so daß es sich erübrigt auf einzelnes einzugehen. Nur eines will beachtet sein, nämlich der Vorwurf, daß Haller sich über das Nachdruckverbot für Boerhaave-Vorlesungen, also über einen ausdrücklich vom Lehrer geäußerten Wunsch, hinweggesetzt und es dadurch an der schuldigen Pietät ihm gegenüber habe fehlen lassen. Denn dieser Vorwurf wird nicht nur von Noortwyck und, wie später zu zeigen sein wird, auch von van Swieten erhoben; auch Albinus bringt ihn in seinen 1754 erschienenen Annotationes academicae ${ }^{38}$ unter anderen Anschuldigungen neuerdings und mit Nachdruck vor. So sehen wir die Kluft zwischen Haller und dem Stammkreis der Leidener Boerhaave-Schüler breiter werden und Haller im Zwist um die Praelectiones, letztlich aber wegen seiner freieren Auffassung des Pietätsverhältnisses zu Boerhaave, immer mehr in Gegensatz zum Leidener Kreis geraten, von dem selbst sein sonst gutes Verhältnis ${ }^{39}$ zu dem Nachfolger auf der Boerhaave-Lehrkanzel und Verfasser des führenden Pathologie-Lehrbuches der Zeit, Hienonymus Davio GaUb (1705-1780), nicht unberührt bleibt. Dies alles muß Haller nicht nur persönlich geschmerzt haben; schließlich war Leiden mit seinen führenden Männern, Albinus und Gaub, und seiner Pflanzschule in Wien mit van Swieten und de Haen auch nach dem Tode Boerhaaves eine wissenschaftliche Macht von weithin ausstrahlender Wirkung.

Der im Frühsommer 1748 von Haller unternommene Versuch, in persönlicher Fühlungnahme mit van Swieten die leidigen Differenzen aus der Welt zu schaffen, stand von vorneherein unter keinem guten Stern. Denn zu ebenderselben Zeit ließ der Anonymus der Bibliothèque raisonnée in dieser ${ }^{40}$ seine Replique auf das Libell Noortwycks erscheinen, in der er, offenbar aus dem Wunsche zu überzeugen, nicht nur sein BoerhaavePortrait noch schärfer profilierte, sondern auch in eine Einzelargumentation gegen van Swieten eintrat. Dieser konnte dort nicht nur lesen, wie veraltet seine in den ersten zwei Bänden vorgetragenen Boerhaave-Dogmen

38 B.S. Albivus, Annotationes academicae, Lugd. Bat. 1754, Lib. III, Ad cap. XII, p. 84 ss.; dazu Hallers Entgegnung in der Vorrede zum 4. Band der Elementa, S. V-XXXII.

39 Haller hatte ihm 1744 den fünften Band der Praelectiones gewidmet und sein Name steht auch vor der Ausgabe der lateinischen Briefe an Haller, Bern 1773. Vgl. L. Hirzel, a.a. O., S. XXXII, Anmerkung 2; 1759 aber schrieb HALLER über Gaub an Gesner (Sigerist, Nr. 214, S. 285): «... cujus quas scribit voculae nescio quid habent invidiosi» und 1771 (Sigerist, Nr. 427, S. 452): «Mortuo Albino nunc audebit amicitiam fateri.» 40 Bibl. rais. 40 (1748) 205-26, 454-68. 
waren wie beispielsweise seine Gefäßeinteilung in Blut-, seröse und lymphatische Gefäße oder das in den $\S \S 38 \mathrm{ff}$. gebrachte System vom Faseraufbau des Körpers ${ }^{41}$, seine Entzündungslehre, seine Ablehnung der Chinarinde usw.; er konnte dort auch das freimütige Bekenntnis über Boerhaave finden: "C'est moi, qui ai douté que Boerhaave ait été infaillible, et qui, en lui prodiguant les Epithètes les plus honorables, ai pris la liberté de dire qu'il y avoit des hypothèses dans ses ouvrages; qu'il n'avoit pas été anatomiste dans toute l'étendue de ce titre, et qu'en un mot ce grand-homme a été mortel.»Was ihm, van Swieten, ein Leben lang das Orakel gewesen war, Boerhaave, für den Anonymus der Bibliothèque raisonée blieb er zwar ein grand-homme, aber er war kein infallibler mehr, sondern ein Sterblicher geworden.

Der langjährige Göttinger Hausgenosse, Schüler und Biograph Hallers, Johann Georg Zimmermann (1728-1804) ${ }^{42}$, läßt in der Lebensbeschreibung seines Lehrers (S. $220 \mathrm{ff}$.) keinen Zweifel, daß Haller selbst der Anonymus der Bibliothèque raisonnée gewesen ist, und das wurde auch in der Folge so beispielsweise in der noch immer grundlegenden Haller-Biographie Ludwig Hirzels (S. CCLIII) als gesichert betrachtet. Diese Überzeugung der Haller-Biographen erleidet auch dadurch keinen Eintrag, daß Haller selbst in seinem Brief an van Swieten vom 26. Mai $1748^{43}$ sein Inkognito nicht lüftet und in bezug auf den Artikelschreiber van Swieten gegenüber nur von dem "anonymus auctor in Bibliothèque Raisonée» spricht. Diese Zurückhaltung gebot einfach der Zweck des Briefes, der auf eine Beilegung des Streites, nicht aber auf dessen Verschärfung abzielte, wie sie notwendigerweise Hallers Einbekenntnis der Autorschaft zur Folge gehabt hätte. Die Richtigkeit von Zimmermanns Angabe wird auch durch das Material bestätigt, das die sehr sorgfältig gearbeitete Münchener Dissertation FrITZ

41 VAN Swieten nahm wie Boerhaave als einfachstes Bauelement die Faser-Fibra an. Durch Aneinanderlegen von mehreren Fasern in der Längsrichtung entsteht die Membran, durch deren Einrollung das kleinste Gefäß und so fort immer größere bis zur Aorta. Diese als das größte Gefäß besteht demnach aus Membranen, die aus kleineren Gefäßen zusammengesetzt sind. Dagegen richtet sich die Kritik in der Bibl. rais. 40 (1748) 464f. Über die mechanistisch-mathematische Ausprägung, die die Faserlehre im BoerhaaveKreis fand, vgl. A. Berg, Die Lehre von der Faser als Form- und Funktionselement des Organismus, Virch. Arch.f. path. Anat. 309 (1942) $407 \mathrm{ff}$ - HAller hat diese mechanistische Form der Faserlehre auch bei einem anderen Boerhaave-Schüler, bei J. F. ScHreIBER, kritisiert: «... haeret nimis ad Boerhaavianas theorias» (Sigerist, Nr. 197, S. $273 \mathrm{f}$.).

42 Dazu vgl. die verschiedenen Gedenkaufsätze in der Schweiz. Med. Wschr. 9 (1928) Nr.49.

43 Neues Magazin für Ärzte 2, (1780) 3. St., S. $207 \mathrm{f}$. 
MeIERS $^{44} 1915$ zur Abfassungsgeschichte der Zimmermannschen HallerBiographie beibrachte. Da erfahren wir (S. $23 \mathrm{ff}$.), daß Haller selbst seinem Biographen nicht nur reichlich Unterlagen zur Verfügung stellte, seine Tagebücher, verschiedene "mémoires», Kopien von Aufsätzen und Bücher, sondern daß sich unter den letzten eben auch jener erste Teil des 33. Bandes der Bibliothèque raisonnée befand, in dem der den Streit auslösende Artikel Hallers abgedruckt war. Außerdem ließ sich Haller die druckfertigen Bogen von Zimmermanns Manuskript zur Korrektur zuschicken. Wäre er also tatsächlich nicht der Anonymus der Bibliothèque raisonée gewesen, so hätte er beim Korrekturenlesen sicherlich und zumal bei der Bedeutung der Sache und der Persönlichkeiten die Gelegenheit wahrgenommen, Zimmermanns Manuskript dementsprechend richtigzustellen.

Es wäre nun weit gefehlt, nach heutigen Maßstäben die Anonymität Hallers als Kritikers mit einem negativen Vorzeichen zu versehen. In der Zeit Hallers war es in den angesehensten Journalen das übliche, Anzeigen und Kritiken unsigniert zu bringen. Und das gehörte auch zu den strikten Gepflogenheiten der seit 1742 erscheinenden Bibliothèque raisonée, der Haller als Mitarbeiter angehörte ${ }^{45}$. Die Redaktion dieser Zeitschrift begündet in der Vorrede ${ }^{46}$ ihre Gepflogenheit damit, daß sie in der Anonymität die beste Gewähr für eine möglichst objektive Kritik erblicke. Haller selbst teilte diese Ansicht. In der Vorrede zum zweiten Band der Elementa äußert er sich darüber in bezug auf eine von ihm ebenfalls anonym gebrachte Bemerkung über Albinus: «Ich merkte dieses nicht in meinem Namen, sondern als ein Schriftsteller eines Tagebuches an. Denn man schreibt anders in Tagebüchern, anders in unserem Namen, indem man an erstern Orte ein öffentliches und von aller eigenen oder fremden Liebe abgesondertes Geschäfte auf sich nimmt, und in so fern etwas freiere Hände behält, daß wir von uns selbst nicht nur reden können, sondern auch bisweilen sogar müssen.»

Aus dieser Auffassung Hallers folgt, daß wir sein nicht signiertes Boerhaave-Bild aus der Bibliothèque raisonnée nicht weniger authentisch und ihm zugehörig zu nehmen haben als seine bekannten Bekenntnisse zu Boerhaave aus den Tagebüchern und Vorreden zu seinen Werken wie beispiels-

44 F.MeIER, Beiträge zur Biographie Albrecht von Hallers, Phil. Diss. München 1915.

45 J.G.ZimmermanN, a. a. O., S. $183 \mathrm{f}$.

16 F.MeIER, a. a.O., S. 74, Anmerkung 15. 
weise die viel zitierte ergreifende Stelle der Dedikatio des ersten Bandes der Praelectiones, in der er der Hoffnung Ausdruck gibt, seinen Schülern noch als Greis von seiner Liebe zu dem Lehrer künden zu können: «Excitabat utique me, amor Praeceptoris; quem et cum eo Johannem Bernoullium me audiisse, senex olim, si quidem annos Deus concedet, non absque animi innocente elatione narrabo discipulis.»

Man könnte nun meinen, daß der signierende Haller anderes über Boerhaave sage als der nicht signierende, daß die angeführte und andere Stellen aus Hallers Werken in Widerspruch stünden zu dem aufgeklärten Boerhaave-Bild der Bibliothèque raisonnée. Dem ist aber nicht so. Vielmehr ergänzen sich die verschiedenen Stellen und auch die früher in Anmerkung 14 angeführten aus den Briefen an Gesner zu dem ganzen, dem wahren Boerhaave-Bild Hallers, das man am besten so umschreiben wird: Boerhaave, der Lehrer, ist immer der grand-homme und bleibt trotz allen in der Bibliothèque raisonnée aufgedeckten Mängeln und Irrtümern nicht weniger verehrungswürdig für den kritisierenden Schüler in Göttingen als er es für den gläubig auf seine Worte schwörenden in Wien war.

Der Marburger Medizinprofessor Ernst Gottrfied Baldinger (1738 bis 1814) ist immer ein geschickter Redaktor seiner ärztlichen Magazine und Journale gewesen. Er hat 1780 gewußt, daß drei Jahre nach dem Tode Hallers und acht nach jenem van Swietens die ärztliche Welt nichts so sehr interessierte als authentisch zu erfahren, warum die beiden größten Boerhaave-Schüler ein Leben lang einander fremd gegenüberstanden. Und tatsächlich war Baldinger in der Lage, die Leser seines Neuen Magazins darüber durch die Veröffentlichung des originalen Haller-van Swieten-Briefwechsels aus dem Jahre 1748 aufzuklären ${ }^{47}$. Sein Wiener Korrespondent, von Retzer, hatte ihm dafür die Unterlagen verschafft.

Der erste Brief Hallers trägt das Datum des 26. Mai 1748. Es ist jener, in dem Haller von der den ganzen Zwist auslösenden Bemerkung Werlhofs, leicht retouchierend, als «einem allzu witzigen Urteil», einem «dicacius iudicium felicissimi medici et mihi optime noti» spricht und sich dabei gleichzeitig mit der Frage distanziert: «Quid ea ad me?» Denn der Brief ist sichtlich von dem Wunsche nach einer Verständigung bestimmt und von der Sorge getragen, eine Ausweitung des Konfliktes zu einem regelrechten Bellum literarium könnte Platz greifen. An solchen aber hatte Haller zu eben dieser Zeit nachgerade genug. Die Fehde mit Hamberger war

${ }^{47}$ Neues Magazin für Ärzte 2 (1780) 3. St., S. 206-15. 
seit 1746 in vollem Gange ${ }^{48}$, seit 1747 auch die Auseinandersetzung mit Julian Offray de la Mettrie ${ }^{49}$, der Gegensatz zu Linné ${ }^{50}$ machte sich bereits bemerkbar und eben jetzt 1748 drang auch der Streit mit seinem Schüler und Göttinger Hausgenossen, Johann JAcoв Huber (1707-1778) ${ }^{51}$, in die Öffentlichkeit, während jener mit dem Göttinger Anatomen JoHANN GotTfried Brendel (1712-1758) dank dem Geschick des trefflichen Universitätskurators MüNCHHAUSEN beigelegt werden konnte ${ }^{52}$. So versteht man es, daß sich Haller versichern wollte, nicht auch noch in Wien einen machtvollen Feind zu haben.

Im Eingang seines Briefes erinnert daher Haller van Swieten an all das, was sie verbindet: an die gemeinsamen Jahre in Leiden, an den gemeinsamen Lehrer Boerhaave und auch daran, daß er van Swieten in seinen Werken stets zitiere, während er Gleiches von dessen Seite bisher vermisse. Dieser Umstand hat Haller sichtlich gekränkt. Er war eben viel mehr von dem Meinen und Glauben anderer über ihn beeindruckbar als van Swieten, der von sich in seinem Brief an Haller in gelassener Selbstsicherheit aussagen konnte: «quid alii faciant, mea nihil refert.»Van Swieten fand in sich und seinem Glauben jene "tranquillitas animi», jene Ruhe des Gemüts, deren Nichtstörbarkeit weder durch den Witz eines Werlhof noch durch die Kritik eines Anonymus er Haller gegenüber so oft sich rühmt. Dieser dagegen muß mit der Unrast und «unruhigen Werksucht » ${ }^{53}$ seines kämpferischen Gelehrtentemperaments selbstquälerisch hadern, und es ist ergreifend zu erfahren, wie er seinen Gott gerade um das bittet, was van

48 Vgl. die in Anmerkung 36 genannte Literatur.

49 Einzelheiten bei L. Hirzel a. a. O., S. CCLIVff.; STEPHEN D'Irsay, Albrecht von Haller, Arbeiten aus dem Institut für Geschichte der Medizin an der Universität Leipzig, Band 1, Leipzig 1930, S. 28f.; R. SAussure, Haller and la Mettrie, J. Hist. Med. 4 (1949) 431-49.

${ }^{50}$ E. Fischer, Hallers Beziehungen zu den Naturforschern seiner Zeit, speziell zu Linné, Mitteilungen der Naturforschenden Gesellschaft Bern aus dem Jahr 1908, Bern 1909, S. 145-72; H. Goerke, Die Beziehungen Hallers zu Linné, Sudhoffs Arch. Gesch. Med. 38 (1954) 367-77; H. Zoller, Albrecht von Haller und seine Beziehungen zu Carl von Linné, Literaturbeilage der Neuen Zürcher Zeitung vom 19. Oktober 1958, Blatt 5, Nr. 3025 (73). Die Kenntnis dieses ausgezeichneten Aufsatzes verdanke ich der Güte Professor H. Fischers.

${ }^{51}$ Darüber L. Hirzel, a. a. O., S. CCLXII ff.

52 Götz von Selle, Die Georg-August-Universität zu Göttingen. 1737-1937, Göttingen 1937, S. 73.

${ }^{53}$ Albrecht von Hallers Tagebuch seiner Beobachtungen über Schriftsteller und über sich selbst, 2. Th., Bern 1787, S. 253. Eintragung vom 26. Juli 1741. 
Swieten in so selbstverständlicher Weise besa $\beta^{54}$ : «Gieb mir Gelassenheit und christliche Liebe, und Ruhe des Gemüths.»

Und nun fühlt sich dieser unruhige und leicht beunruhbare Gelehrte durch die anscheinende Nichtbeachtung seiner Werke durch den ehemaligen Mitschüler verletzt. Ja, dieses Gefühl schien sogar eine Bestätigung gefunden zu haben durch die Nachricht eines belgischen Korrespondenten, der ganz gewiß wissen will, daß van Swieten Hallers Werke tatsächlich mißfielen. Außerdem, so schreibt Haller weiter an van Swieten, habe dieser Korrespondent mitgeteilt, daß van Swieten, ungehalten über den Artikel in der Bibliothèque raisonnée mit seinem «allzu witzigen Urteil», nicht nur der Initiator des Noortwyckschen Libells gewesen, sondern eben jetzt auch daran sei, eine Kritik gegen Hallers gesamtes Werk starten zu lassen. Er, Haller, betrachte es nicht unter seiner Würde, ihn, van Swieten, zu bitten, davon Abstand zu nehmen und der Welt nicht das Schauspiel der streitenden Boerhaave-Schüler zu bieten, sondern von allen Kontroversen zu lassen. Aber zwiespältig und leicht sich erhitzend, wie eben der Schüler in Göttingen ist, wird er getrieben, diesen wahrhaft versöhnlichen Worten jetzt auch noch andere zuzufügen, daß nämlich er und seine Freunde, sollte dieser Befriedungsversuch scheitern, nicht nur sich selbst zu verteidigen, sondern auch die anderen anzugreifen wüßten.

Hart ist die Antwort van Swietens in Inhalt und Form. Haller hat dieses Urteil an den Anfang seines Antwortschreibens gestellt. Und in der Tat! Zum ersten tut van Swieten die Anzeige der Bibliothèque raisonnée als einen von verlegerischen Interessen inspirierten und bestimmten Reklameakt $\mathbf{a b}^{55}$. Über den Verfasser dieser Anzeige: alle Gutgesinnten wären über ihn indigniert, weil er Boerhaave, den Lehrer, als einen «anatomes imperitus et hypothesium conditor et ornator» hingestellt habe. Ein Schmäher, ein «calumniator» in den Augen der mit wahrer Pietät erfüllten BoerhaaveSchüler. Und zu Haller: der vir celeberrimus möge sich nicht gekränkt fühlen. Denn er, van Swieten, habe dessen Werke keineswegs aus Verachtung nicht gelesen, sondern aus Zeitmangel. Im übrigen habe er mit dem Noortwyckschen Libell nichts zu tun, habe es bis jetzt überhaupt nicht gesehen und denke nicht im entferntesten daran, mit ihm, Haller, ein Bellum literarium zu führen.

54 A. v. Haller, a.a. O., S. 265. Eintragung vom 20. Juli 1746.

55 Van Swieten bezieht sich hier auf den Umstand, daß derselbe Verlag Wetstein, in dem die Bibliothèque raisonnée seit 1742 erschien, 1739 auch eine Auflage von Hallers Boerhaave-Kommentar herausgebracht hatte. 
Tatsächlich lehnte van Swieten solche Gelehrtenstreite grundsätzlich ab und ging in seiner Ablehnung sogar so weit, auch andere daran zu hindern. Energisch tat er dies im Streit seines Mitarbeiters de Haen mit Haller, als er 1766 in seiner Eigenschaft als Zensor die Veröffentlichung neuerlicher Stellungnahmen seines streitbaren Freundes ${ }^{56}$ hinsichtlich der Irritabilitätslehre verhinderte ${ }^{57}$. Den Standpunkt, den er persönlich einnahm, formulierte er anläßlich des Schierlingstreites 1761 de Haen gegenüber folgendermaßen ${ }^{58}$ : «J'avoue, que je suis ennemi de toute dispute, on m'a attaqué plus d'une fois, et j'ai laissé faire ${ }^{59}$ : je ne me soucie pas beaucoup de ce qu'il Vous plait nommer reputation. Je sens beaucoup d'indolence sur cet article et je m'en trouve bien. Ne vous mettez pas en harnois pour me defendre, si l'on dit du mal de moi, car je crois être trop paresseux pour le faire moi même, parceque je n'ai pas grande opinion de moi, et l'exercise journalier de mon art me confirme de plus en plus dans cette pensée.»

Seine Bescheidenheit ebenso wie die Einsicht in das stets Unvollkommenbleibende des eigenen Wollens und Meinens waren es, die van Swieten von dem Anspruche abhielten, dieses Eigene als das Alleingültige anderen gegenüber zu behaupten und zu verteidigen. Auch in der Befolgung dieses Grundsatzes erweist sich der Schüler in Wien, van Swieten, als der gesinnungsgetreue Nachfolger des Leidener Meisters, an dessen gleiche Maxime ein anderer großer Boerhaave-Schüler in Uppsala, CarL von Linné (1707 bis 1778), den in Göttingen gemahnt ${ }^{60}$ : «Unser Spiegel, Boerhaave, pflegte niemals zu erwidern. Ich erinnere mich seines Ausspruches an mich: niemals sollst du auf Apologien entgegnen. Dies sollst du mir versprechen. Ich versprach es und ich bin dabei sehr gut gefahren.»

Aber Haller drängt sein Forschertemperament, auch hier eigene Wege zu gehen, über die gemeinsame Schulmaxime hinwegzuschreiten und dem van

${ }_{56} \mathrm{Zu}$ dessen Naturell fand Haller noch eher Zugang als zu dem van Swietens. Am 9.Februar 1759 äußerte er sich über die beiden Gelehrten zu seinem vertrauten Freunde Gesner (Sigerist, Nr. 219, S. 293): «A. de Haen... multo minus ille senilis ingenii quam quidem Swietenius, minusque invidi. »Vgl. auch das ähnliche Urteil bei Sigerist, Nr. 217, S. 291.

57 Darüber berichtet de Haen in seinem Brief vom 28. März 1768 an Haller. Epistolarum ab eruditis viris ad Alb. Hallerum scriptarum, Vol. V, Bernae 1774, Nr. 272, p. 332 s.

58 Brief van Swietens an de Haen vom 10. September 1761. Abgedruckt in E. G. BaLdinger Neues Magazin für Ärzte 2 (1780) 1. St., S. 49.

${ }^{59}$ Von mir hervorgehoben.

${ }^{60}$ Epistolarum ab eruditis viris ..., Vol. II, Nr. 390, p. 409. Brief Linnés an Haller vom 13. September 1748. Über Boerhaave und Linné vgl. G. A. Lindeboom, Janus 47 (1957) 264-274. 
Swietenschen Prinzip des «laisser faire» im Sinne Heraklits die Überzeugung entgegenzusetzen, daß der Krieg der Vater aller Dinge sei. In der "Vorrede zum I. Theile der Allgemeinen Historie der Natur ${ }^{61}$ bekennt sich Haller zu diesem Prinzip als dem seinen: «Streitende Secten sind wie Stahl und Feuersteine: sie zeugen zwar Feuer, aber auch Licht dabey, uns zu erleuchten ... Der Preiß ist die Ehre des Rechthabens, und das gemeine Beste genießt die Früchte des Bestrebens der Streitenden.» Gerade der Streit mit Hamberger über den Atmungsmechanismus hat Haller die Fruchtbarkeit dieses seines Prinzips bestätigt ${ }^{62}$ : "Ich wurde genöthiget, neue Versuche zu machen, und diese öfters zu wiederholen, und fand nicht nur die Wahrheit dessen, das ich vertheidigte, ich fand auch neue Gründe dazu, und überzeugte mich, daß kein Grund mehr bleiben könnte, warum man an einer Lehre zweifeln sollte, deren Richtigkeit ich mit Augen an so vielen Thieren gesehen hatte.»

So verstehen wir, daß Haller nicht nur von der Richtigkeit und Nützlichkeit seines agonalen Prinzips überzeugt war, sondern daß er dieses geradezu als ein notwendiges Desiderat einer vorwärtsstrebenden, fruchtbaren und befruchtenden Forschung ansah. Wir verstehen aber auch, daß sich mit dieser Überzeugung der Gegensatz zu van Swieten nur noch mehr vertiefen mußte. Dort der edle Wettstreit der Meinungen, den die Unruhe einer kämpferisch-kritischen Forschernatur zum heuristischen Prinzip erhob, hier die tranquillitas animi, die selbst noch in ihrem «laisser faire» das gläubige Beharren als die ihr gemäße Norm setzt. Grundsätzliches stößt hier hart aufeinander und spiegelt in neuer Abwandlung den Grundkonflikt dieser beiden Männer wider in ihrem Verhältnis zu Boerhaave. Denn während Haller in seinem Brief erklärt, im Falle des Scheiterns seines Verständigungsversuches van Swieten und die Seinen angreifen zu wollen, beharrt dieser auch hier auf seinem vom Lehrer überkommenen Grundsatz, den Angriff nicht zu erwidern. Dies solle seine Form der Erwiderung sein. Ruhigen Sinnes (tranquilla mente) werde er den Angriff erwarten.

Das gegenseitige Meinen und Wollen hatte sich im Grundsätzlichen festgefahren. Oder ist es nicht vielmehr so, daß dessen Grenzen und Möglichkeiten schicksalhaft in der wesensmäßigen Verschiedenheit der beiden Männer bereits vorgebildet lagen, bevor es überhaupt zu Erklärungen und zum Konflikte kam? Dieser aber mußte sich noch weiter verschärfen, als van

61 Sammlung kleiner Hallerischer Schriften, 1. Th., Bern 1772, S. 70.

62 A.v.Haller, a. a. O., S. 69. 
Swieten in seinem Antwortschreiben unmißverständliche Kritik an Hallers Vorgehen bei der Herausgabe der Boerhaave-Vorlesungen über die Institutionen übte. Er, van Swieten, habe sich ein Gewissen daraus gemacht, «nach dem Tode Boerhaavens nicht das zu tun, was, wie (er) wußte, dem großen Manne während seiner Lebzeiten so sehr mißfallen hat, nämlich: Flicken (centones) aus den Diktaten (wie man es nennt) verschiedener Jahre zusammenzuscheren (corradere) und unter dem Namen des Lehrers selbst herauszugeben.»

Diese so scharf formulierte Kritik van Swietens hat Haller tief verletzt, und fast wundert man sich, daß er noch einmal nach Wien schrieb. Wieviel muß ihm an dem Manne dort gelegen sein, wenn er in seinem Brief vom 7.Juli 1748 immer noch maßvoll erwidert: «Nicht zusammengescherte Skripten (corrasis codicibus) habe ich benutzt, sondern die meinen und die des Johannes Gesner und des B(ernhard) Feldmann, die hervorragend gelehrte Männer sind.»

Tatsächlich hatte sich Haller sehr viel Mühe gegeben, die Lücken seines Skriptums möglichst autorgetreu zu ergänzen. Denn solche Lücken waren da, da er ja nicht wie van Swieten tachygraphieren konnte, sondern offenbar nur die sinntragenden Worte mitschrieb. Da erwies es sich für ihn sehr nützlich, und er hat dies auch in den Vorreden bzw. Dedikationen zum ersten und vierten Band der Praelectiones ebenso wie in seiner Anzeige der Bibliothèque raisonnée dankbarst anerkannt, daß die obengenannten Freunde ihm ihre Kolleghefte zur Verfügung stellten. Natürlich gelangte Haller auch mit diesen Hilfen nicht zu einer wortwörlichen Rekonstruktion des Boerhaave-Textes, wie ihn van Swieten in dem Schatz seiner Tachygramme besaß. Das hat der holländische Medizinhistoriker, E.C.van LeErsum ${ }^{63}$, für die Hallersche Ausgabe der Introductio in Praxin clinicam Boerhaaves erwiesen, indem er diese Ausgabe mit dem entsprechenden und von ihm dechiffrierten Tachygramm van Swietens verglich. Darnach ist der von Haller abgedruckte Text als eine, wenn auch nicht wörtliche, so doch im wesentlichen sinngetreue Wiedergabe der genannten Boerhaave-Vorlesung anzusprechen.

Es liegt nahe, eine solch sinngetreue Wiedergabe durch Haller auch für die noch nicht mit der van Swietenschen Mitschrift verglichenen Vorlesungen über die Institutionen anzunehmen. Ist das richtig, dann läßt sich Hallers Arbeit an ihnen, ganz abgesehen von dem hohen Eigenwert seines

63 E. C. van Leersum (s. Anmerkung 5), S. 324 ff. 
Kommentars, nicht in das Bild von dem «Zusammenscheren von Flicken» (centones corradere) einordnen; indem aber van Swieten dies tat, dürfte er mit diesem in seinem Übermaß an Pietät für den Lehrer doch zu weit gegangen sein.

So hat der von Haller in bester Absicht eingeleitete Briefwechsel mit van Swieten nicht nur keine Annäherung der Standpunkte gebracht; er hat diese vielmehr auf allen Linien versteift, die bestehenden Gegensätze nur noch schärfer hervortreten lassen und die beiden Männer gegeneinander verhärtet. Was Haller bei einer solchen Lage der Dinge künftig für beide Teile noch erhoffen kann, drückt er am Schlusse seines zweiten Briefes in dem Wunsche nach einem achtungsvollen Respektieren der beiderseitigen wissenschaftlichen Leistungen aus, wobei es ihm im besonderen darauf ankäme, «ut constat aliquod Tuum de me iudicium».

Dies ist denn in der Folge auch von seiten van Swietens geschehen. In den seit 1753 erscheinenden Bänden seiner Kommentare berücksichtigt und zitiert er eine Reihe von Hallers Arbeiten; man kann sie im Catalogus auctorum des fünften Bandes zusammengestellt finden. Aber bereits im dritten, 1753 erschienenen Band (§ 1060, p. 357) spricht van Swieten von Haller, ohne daß dies der Zusammenhang notwendigerweise erforderte, als von dem «celeberrimus Haller propriis laboribus de republica literaria optime meritus ». Das mag der rasche Leser als eine der üblichen Gelehrtenapostrophierungen der Zeit nehmen; der aufmerksame aber, der die Zusammenhänge kennt, um deren Klärung sich diese Untersuchung bemühte, wird in dieser Apostrophierung genau jenes honorificum iudicium erkennen und es als versöhnliche Geste van Swietens werten, auf die es Haller in seinem letzten Briefe vom 7.Juli 1748 ankam.

In den nach der Auseinandersetzung mit Haller erscheinenden Bänden des van Swietenschen Werkes läßt sich aber auch noch ein Anderes, nicht weniger Bedeutungsvolles beobachten. Auch van Swieten wird jetzt immer freier, immer mehr er selbst. Indem er eigene Beobachtungen, Erfahrungen und eigene Meinungen, die vielfach von denen des Lehrers abweichen, in seinen Kommentaren in zunehmendem Maß vorträgt, wird der bedingungslose Boerhaave-Dogmatiker der Vierzigerjahre, für den Boerhaave schlechtweg das Orakel war, zum Boerhaave-Eklektiker der Reife- und Spätzeit. Er wird nun wirklich und im eigensten Sinne zu dem, was er der Titelgebung nach schon vom ersten, 1742 erschienenen Band sein sollte: zum Autor seiner Kommentare. Der letzte, in seinem Todesjahr 1772 erschienene Band ist ganz sein Werk. Zwar geht van Swieten immer noch vom Boer- 
haave-Aphorismus aus; aber was er selbst und Eigenstes über epidemische Krankheiten, Steinleiden, Lues, Rachitis und Rheumatismus zu sagen hat, ist soviel, daß der zugrundeliegende Boerhaave-Aphorismus mehr oder weniger als ein historisches Requisit, immer aber als ein achtungsvoll behandeltes erscheint. Wir wissen nicht, ob und inwieweit Hallers Kritik an dieser Entwicklung beteiligt war oder auch nur eine ausgelöst hat, die in der Eigengesetzlichkeit von van Swietens Persönlichkeit vorgegeben lag. So müssen wir uns mit der rein chronologischen Feststellung begnügen, daß diese Entwicklung nach der Auseinandersetzung mit Haller Platz gegriffen hat. Eines aber können wir in jedem Falle sagen, daß van Swieten durch seine Entwicklung vom Boerhaave-Dogmatiker zum Boerhaave-Eklektiker viel von dem bestätigte, worum es Haller in der Auseinandersetzung der Bibliothèque raisonnée gegangen ist. 